Original paper

\title{
Predictive power of Model for End-Stage Liver Disease and Child-Turcotte-Pugh score for mortality in cirrhotic patients
}

\author{
Damian Piotrowski ${ }^{1}$, Anna Sączewska-Piotrowska² Jerzy Jaroszewicz'1 Anna Boroń-Kaczmarska' \\ ${ }^{1}$ Medical University of Silesia in Katowice, Poland \\ 2University of Economics in Katowice, Poland
}

\begin{abstract}
Aim of the study: To assess the performance of Child-Turcotte-Pugh (CTP) and Model for End-Stage Liver Disease (MELD) scores' kinetics during hospitalization in predicting in-hospital mortality in patients with liver cirrhosis.

Material and methods: One hundred and seventy-four cases of hospitalized liver cirrhosis patients were selected. The diagnosis of cirrhosis was made based on clinical, biochemical, ultrasonic, histological, and endoscopic findings and results. CTP and MELD scores at admission and $\triangle C T P$ and $\triangle M E L D$ were calculated. Univariate and multivariate logistic regression and receiver-operating characteristic (ROC) curve analysis were performed. In the models, odds ratios (ORs) and 95\% confidence intervals (Cls) were calculated. The area under the ROC curve (AUC) was used to measure the accuracy. For the optimal cutoff point, sensitivity (SE), specificity (SP), positive predictive value (PPV), and negative predictive value (NPV) were calculated. The Kaplan-Meier method was used to construct survival curves, and the log-rank test was used to compare time to death, with respect to MELD and CTP categories.

Results: Among the assessed scores, the highest area under the ROC curve (AUC) in univariate logistic regression analysis was calculated for $\triangle M E L D \geqslant 1$, followed by $\triangle C T P \geqslant 1, C T P>8$, and MELD $>17$. Based on the selected criteria, multivariate models were created that were characterized by an outstanding ability to predict the in-hospital mortality.

Conclusions: In-hospital mortality is relatively high in patients with liver cirrhosis. The combination of CTP and MELD scoring methods, combined with their kinetics, allows for the prediction of short-term mortality.
\end{abstract}

Key words: liver cirrhosis, mortality, scoring models.

\section{Address for correspondence}

Damian Piotrowski, MD, Medical University of Silesia in Katowice, Aleja Legionów 49, 41-902 Bytom, e-mail: dpiotrowski@sum.edu.pl

\section{Introduction}

Liver damage may be caused by various factors, including but not limited to viral infections, toxic damage, and metabolic diseases. Liver cirrhosis is the final stage of damage [1].

There are two score models that are commonly used to assess the severity of liver dysfunction. The first is the Child-Turcotte-Pugh (CTP) score, initially proposed by Child and Turcotte and later modified by Pugh et al. [2]. The second is the Model for End-Stage
Liver Disease (MELD) [3]. These two score models are widely used to predict the outcomes of cirrhotic patients.

The aim of this paper is to present results from an analysis of hospitalizations of cirrhotic patients in the Department of Infectious Diseases at the Medical University of Silesia, confirm the predictive values of CTP and MELD scores for mortality in cirrhotic patients, and determine whether changes in CTP and MELD scores during hospitalization can be used in the assessment of prognosis in patients with liver cirrhosis. 


\section{Material and methods}

\section{Study design}

There were 174 hospitalizations between 2004 and 2016 in the Department of Infectious Diseases at the Medical University of Silesia, which were selected for inclusion in this case-control study. The only inclusion criterion was the diagnosis of liver cirrhosis. The diagnosis of cirrhosis was confirmed based on clinical, biochemical, ultrasonic, histological, and endoscopic findings and results. The total number of patients included was 138 . Most of them were hospitalized only once in the study period; however, 14 patients were admitted twice, four were admitted three times, two were admitted four times, and two were admitted five times to the hospital. To compare the mortality, two control groups were selected, and matched for age and sex with respect to the observed patients. The first control group consisted of patients with hepatitis (viral, autoimmune, NASH, or cryptogenic) but not cirrhosis, and the second control group included other hospitalized patients without a diagnosis of liver disease.

The study was approved by the Ethics Committee of the Medical University of Silesia.

\section{Data collection}

After identifying the study and the control groups, the data from hospital discharge cards were analyzed. Outcomes of interest were patient death and discharge. However, laboratory tests were also taken into consideration, especially parameters (both laboratory and clinical) that were deemed crucial for the MELD score and CTP classification.

\section{Prognostic scores}

The CTP score consists of three objective symptoms of liver failure (serum bilirubin and albumin concentration and prothrombin time) and two subjective symptoms (presence of ascites and encephalopathy). The CTP score ranges between 5 and 15 points, wherein three classes are distinguished: class $\mathrm{A}$ (5 to 6 points), class B (7 to 9 points), and class C (10 to 15 points). CTP was calculated as described by Pugh et al. [1].

MELD score is determined by three laboratory test results (serum bilirubin, creatinine concentration, and international normalized ratio - INR). This scoring model is the most commonly used method in the evaluation of patients with liver disease for transplantation. The formula for MELD score is:

$9.57 \times \ln ($ creatinine $)+3.78 \times \ln ($ total bilirubin $)+$ $11.2 \times \ln (\mathrm{INR})+6.43$ where creatinine and bilirubin concentrations are represented in $\mathrm{mg} / \mathrm{dl}$, and the computed score is rounded to the nearest integer. If any of the laboratory parameters are lower than 1.0, the score should be set to 1.0 for input so that the formula will avoid generating a negative score. The maximum MELD score is 40 . Any results higher than 40 are adjusted to 40 [2].

The samples for testing were collected from all patients within 24 hours of admission. To evaluate the changes in CTP and MELD scores, another set of samples was collected within a week after admission.

\section{Statistical methods}

All statistical analyses were calculated using STATISTICA (StatSoft, Inc., Tulsa, OK, USA) [4] and R (R Foundation for Statistical Computing, Vienna, Austria) [5] with gmodels [6], OptimalCutpoints [7], survival [8], and verification (NCAR - Research Applications Laboratory) [9] packages.

Univariate and multivariate logistic regression and receiver-operating characteristic (ROC) curve analysis were performed. In the models, odds ratios (ORs) and 95\% confidence intervals (CIs) were calculated. The factors in the models were deemed significant when the $p$ value was less than 0.05 . The area under the ROC curve (AUC) was used to measure the accuracy. The optimum cutoff point was identified as the closest point on the ROC curve (0.1). For the optimal cutoff point, sensitivity $(\mathrm{SE})$, specificity $(\mathrm{SP})$, positive predictive value (PPV), and negative predictive value (NPV) were calculated. The Kaplan-Meier method was used to construct survival curves, and the log-rank test was used to compare time to death, with respect to MELD and CTP categories.

\section{Results}

\section{Clinical characteristics of patients}

The clinical characteristics of the selected patients are described in Table 1 . The mean age of the participants in the study group was 59.2 years, and $52.9 \%$ were men, with a mean body mass index (BMI) of $27.7 \mathrm{~kg} / \mathrm{m}^{2}$. The mean CTP score in the cirrhotic patients was 8.5 (5.8 and 5.5 in the control groups, respectively) and the mean MELD score was 15.0 (8.8 and 7.0 in the first and second control group, respectively). The increased activity of alanine aminotransferase (ALT) in control group 1 was a result of acute hepatitis B (three cases), acute toxic liver damage (two cases), and exacerbation of autoimmune hepatitis (AIH) (one case). Those six patients were characterized by an ALT activity of $800 \mathrm{U} / 1$ or more. 
Table 1. Characteristics of selected patients

\begin{tabular}{|c|c|c|c|}
\hline & $\begin{array}{l}\text { Liver cirrhosis group } \\
\qquad(n=174)\end{array}$ & $\begin{array}{l}\text { Control group } 1 \text { (hepatitis) } \\
\qquad(n=53)\end{array}$ & $\begin{array}{l}\text { Control group } 2 \text { (no liver damage) } \\
\qquad(n=53)\end{array}$ \\
\hline Age [years] ${ }^{1}$ & $59.2(11.0)$ & $54.7(14.0)$ & $54.0(17.1)$ \\
\hline Males $^{2}$ & $92(52.9 \%)$ & $28(52.8 \%)$ & $28(52.8 \%)$ \\
\hline BMI $\left[\mathrm{kg} / \mathrm{m}^{2}\right]^{1}$ & $27.7(5.4)$ & $27.1(4.7)$ & $26.4(4.8)$ \\
\hline $\operatorname{ALT}[\mathrm{U} / \mathrm{I}]^{1}$ & $91.0(139.6)$ & $200.2(412.2)$ & $42.1(91.3)$ \\
\hline bilirubin $[\mu \mathrm{mol} / /]^{1}$ & 65.9 (93.2) & $52.6(79.3)$ & $9.6(5.4)$ \\
\hline Albumin $\left[\mathrm{g} / \mathrm{l}^{1}\right.$ & $31.6(6.7)$ & $39.0(5.9)$ & $36.8(4.4)$ \\
\hline Creatinine $[\mu \mathrm{mol} / /]^{1}$ & $84.4(47.6)$ & $62.3(14.5)$ & $62.9(12.3)$ \\
\hline INR $^{1}$ & $1.49(1.38)$ & $1.02(0.14)$ & $1.12(0.36)$ \\
\hline CTP $^{1}$ & $8.5(2.2)$ & $5.8(1.2)$ & $5.5(0.7)$ \\
\hline MELD' & $15.0(6.5)$ & $8.8(3.9)$ & $7.0(1.0)$ \\
\hline
\end{tabular}

${ }^{1}$ mean (standard deviation), ${ }^{2}$ number (percentage)

BMI - body mass index; ALT - alanine aminotransferase; CTP - Child-Turcotte-Pugh; INR - international normalized ratio; MELD - Model for End-Stage Liver Disease

The reasons for onset of liver cirrhosis varied; however, most of the analyzed patients were cirrhotic due to viral infections: $\mathrm{HCV}$ infection was the basis of the liver cirrhosis in $57.8 \%$, HBV infection in $18.4 \%$, alcohol liver cirrhosis in $10.3 \%$. The remaining cases of cirrhosis were caused by autoimmune hepatitis, primary biliary cirrhosis, non-alcoholic steatohepatitis or the origin of the disease remained unknown.

Regardless of the etiology, the subjects were divided according to CTP classification (class A 20.2\%, class B 49.1\%, class C 30.7\%) and MELD score (6 to 12 points: $39.1 \%, 13$ to 18 points: $38.4 \%, 19$ to 24 points: $14.5 \%, 25$ to 30 points: $5.1 \%, 31$ points or above: $2.9 \%)$. During the period of all 174 hospitalizations, $20(11.5 \%)$ patients died. There were no deceased patients in either control group (20 vs. $0, p=0.005)$.

Whether disease progress affects mortality was also taken into consideration during this study. Nevertheless, whether CTP classification or MELD score was used, the more severe the cirrhosis was, the worse was the prognosis. Among the patients with enough data to evaluate CTP class, 15 died (10 in CTP class C, five in CTP class B). Table 2 shows the mortality in the various CTP classes.

In general, the mortality was higher in patients with more advanced liver disease; however, it is worth mentioning that a significantly higher death rate occurred in patients with decompensated liver disease than in those with compensated cirrhosis (CTP C vs. CTP A $28.6 \%$ vs. $0 \%, p=0.003$ ). The difference between the CTP A and CTP B groups was not statistically significant $(8.9 \%$ vs. $0 \%, p=0.07)$.

As expected, MELD score was found to be associated with mortality. The relevance of MELD score in mortality was observed in patients with a score of 13 or above.

Cirrhotic patients are at risk of death during the course of their disease. The in-hospital mortality was $11.5 \%$ (20 of 174 hospitalizations) in the cirrhotic group, while there was no occurrence of death during hospitalization in either control group $(p=0.005)$. The probability of death increased among cirrhotic patients with the increase in the severity of liver failure.

Although the clinical course of liver cirrhosis varies depending on the etiology, the comparison of mortality in different etiologies was not statistically significant. The mortality of cirrhotic patients was $10 \%$ in $\mathrm{HCV}$-infected patients, $15.6 \%$ in $\mathrm{HBV}$-infected patients $(p=0.3828), 22.2 \%$ in alcoholic liver disease $(p=0.1399)$ and $10 \%$ in the AIH subgroup $(p=0.4184)$. It was taken into consideration whether comorbidities played a role in mortality of cirrhotic patients. Among patients diagnosed with hypertension, diabetes, coronary heart disease and obesity mortality was $5.6 \%$, $5.7 \%, 11.1 \%$ and $11.5 \%$ respectively. It must be noted that no statistical significance was found. Therefore the following analyses of mortality did not take into consideration the etiology of liver cirrhosis or comorbidities.

Among patients with calculated MELD scores, the mortality rate increased from $3.7 \%$ for those with an MELD score $\leq 12$, through $13.2 \%$ (MELD score between 13 and $18, p=0.04$ ) and 35.0\% (MELD score between 19 and 24 ) to $42.6 \%$ (MELD score $\geq 25, p<0.001$ ).

In the logistic regression models, dichotomized variables (MELD score, $\triangle$ MELD, CTP score, and $\triangle \mathrm{CTP}$ ) were used to predict the probability of mortality in cirrhotic patients. 
Table 2. Variables associated with fatality - univariate and multivariate logistic regression analysis

\begin{tabular}{|c|c|c|c|c|}
\hline \multicolumn{5}{|c|}{ Univariate logistic regression analysis } \\
\hline Variable & OR & $95 \% \mathrm{Cl}$ & $p$-value & AUC \\
\hline MELD score > 16 & 3.74 & $1.38-10.13$ & 0.010 & 0.655 \\
\hline MELD score > 17 & 5.17 & $1.90-14.22$ & 0.001 & 0.684 \\
\hline MELD score > 18 & 5.19 & $1.88-14.33$ & 0.002 & 0.675 \\
\hline MELD score > 19 & 4.46 & $1.61-12.36$ & 0.004 & 0.653 \\
\hline MELD score > 20 & 6.24 & 2.18-17.85 & 0.001 & 0.674 \\
\hline MELD score > 21 & 4.76 & $1.59-14.23$ & 0.005 & 0.630 \\
\hline MELD score > 22 & 3.51 & $1.06-11.58$ & 0.040 & 0.585 \\
\hline$\Delta \mathrm{MELD} \geqslant 1$ & 22.80 & $4.37-118.84$ & $<0.001$ & 0.824 \\
\hline$\Delta \mathrm{MELD} \geqslant 2$ & 17.50 & $4.14-73.93$ & $<0.001$ & 0.795 \\
\hline$\Delta \mathrm{MELD} \geqslant 3$ & 15.48 & $3.69-64.86$ & $<0.001$ & 0.769 \\
\hline CTP score $>8$ & 21.54 & $2.72-170.35$ & 0.004 & 0.770 \\
\hline CTP score $>9$ & 5.92 & $1.85-18.99$ & 0.003 & 0.707 \\
\hline CTP score $>10$ & 5.79 & $1.80-18.65$ & 0.003 & 0.668 \\
\hline CTP score > 11 & 20.78 & $5.00-86.38$ & $<0.001$ & 0.713 \\
\hline CTP score > 12 & 24.25 & $4.15-141.56$ & $<0.001$ & 0.657 \\
\hline$\Delta \mathrm{CTP} \geqslant 1$ & 19.43 & $3.38-111.81$ & $<0.001$ & 0.815 \\
\hline$\Delta \mathrm{CTP} \geqslant 2$ & 4.44 & $0.25-77.96$ & 0.307 & 0.538 \\
\hline \multicolumn{5}{|c|}{ Multivariate logistic regression analysis: Model 1} \\
\hline CTP score $>8$ & 74.91 & $4.13-1357.62$ & 0.004 & \multirow{2}{*}{$\begin{aligned} \mathrm{AUC} & =0.908 \\
95 \% \mathrm{Cl} & =0.808-1.008 \\
p & <0.001\end{aligned}$} \\
\hline$\Delta \mathrm{MELD} \geqslant 1$ & 97.32 & $7.95-1191.51$ & $<0.001$ & \\
\hline \multicolumn{5}{|c|}{ Multivariate logistic regression analysis: Model 2} \\
\hline CTP score $>8$ & 74.29 & $2.97-1858.49$ & 0.009 & \multirow{3}{*}{$\begin{aligned} \mathrm{AUC} & =0.941 \\
95 \% \mathrm{Cl} & =0.862-1.020 \\
p & <0.001\end{aligned}$} \\
\hline$\Delta M E L D \geqslant 1$ & 11.90 & $0.53-266.07$ & 0.118 & \\
\hline$\Delta \mathrm{CTP} \geqslant 1$ & 13.20 & $0.63-276.12$ & 0.096 & \\
\hline \multicolumn{5}{|c|}{ Multivariate logistic regression analysis: Model 3} \\
\hline CTP score $>8$ & 64.12 & $2.21-1861.08$ & 0.015 & \multirow{4}{*}{$\begin{aligned} \text { AUC } & =0.956 \\
95 \% \mathrm{Cl} & =0.899-1.014 \\
p & <0.001\end{aligned}$} \\
\hline$\Delta \mathrm{MELD} \geqslant 1$ & 14.57 & $0.53-396.80$ & 0.112 & \\
\hline MELD score > 17 & 2.67 & $0.14-51.11$ & 0.514 & \\
\hline$\Delta \mathrm{CTP} \geqslant 1$ & 15.93 & $0.63-400.08$ & 0.092 & \\
\hline
\end{tabular}

In univariate analysis (Table 2), the best predictive power (using AUC) was found for the following models: MELD score $>17, \triangle$ MELD $\geq 1$, CTP score $>8$, and $\triangle \mathrm{CTP} \geq 1$. Patients with MELD score $>17$ had a significantly higher case-fatality rate than those with MELD score $\leq 17(\mathrm{OR}=5.17,95 \% \mathrm{CI}=1.90-14.22)$. The patient mortality rate was significantly higher in cases with $\triangle \mathrm{MELD} \geq 1$ than in those with $\triangle \mathrm{MELD}<1$ $(\mathrm{OR}=22.80,95 \% \mathrm{CI}=4.37-118.84, p<0.001)$. A CTP score $>8$ was associated with a significantly higher mortality rate $(\mathrm{OR}=21.54,95 \% \mathrm{CI}=2.72-170.35$, $p=0.004)$. Additionally, patients with $\Delta \mathrm{CTP} \geq 1$ had a significantly higher case-fatality rate than those with $\Delta \mathrm{CTP}<1(\mathrm{OR}=19.43,95 \% \mathrm{CI}=3.38-111.81$, $p<0.001)$. The highest AUC estimate was for the model with $\triangle \mathrm{MELD} \geq 1$ (AUC $=0.824,95 \% \mathrm{CI}=0.713$ $0.936, p<0.001$ ), which reflects an excellent ability to discriminate. The lowest AUC estimate was found for models with a MELD score $>17$.

In multivariate analysis with two variables (Table 2, Model 1), the model with CTP score $>8$ and $\triangle \mathrm{MELD} \geq$ 1 had the best ability to predict the probability of mortality (high value of AUC), and the variables were statistically significant. Patients with CTP score $>8$ had 


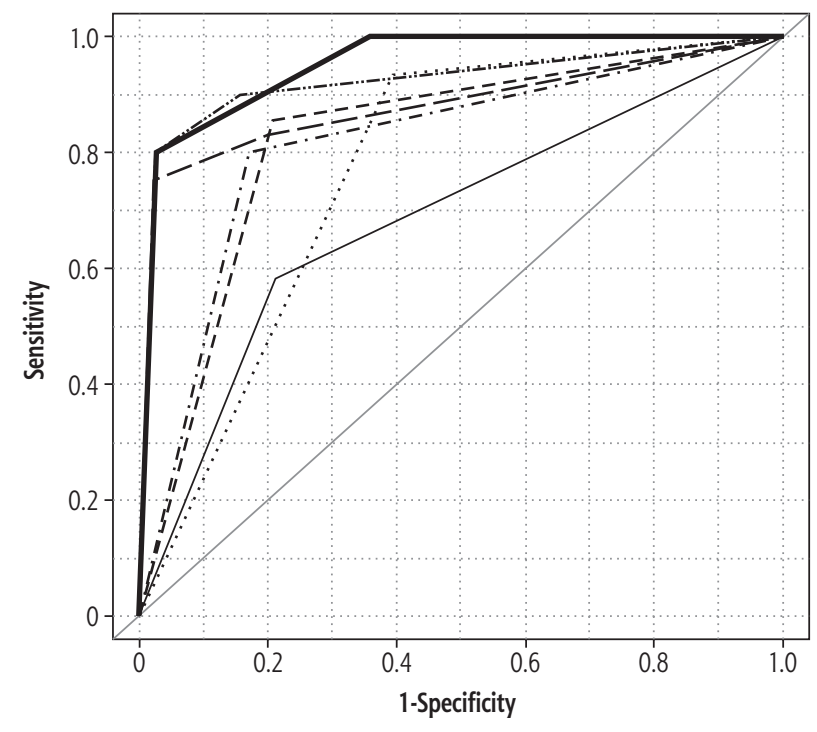

MELD $>17 \quad--\cdot \Delta$ MELD $\geqslant 1 \quad \cdots \cdot C$ CTP $>8 \quad-\cdot-\Delta$ CTP $\geqslant 1$

--2 variables $-\cdots-3$ variables $\longrightarrow$ variables $\quad$ Reference line

Fig. 1. ROC curves of the estimated models

a significantly higher case-fatality risk than those with CTP score $\leq 8(\mathrm{OR}=74.91,95 \% \mathrm{CI}=4.13-1357.62$, $p=0.004)$. Also, patients with $\triangle \mathrm{MELD} \geq 1$ had a significantly higher case-fatality risk than those with $\triangle \mathrm{MELD}<1(\mathrm{OR}=97.32,95 \% \mathrm{CI}=7.95-1191.51$, $p<0.001)$. The optimum cutoff point for predicting mortality risk was 0.9018 with an SE of $75.00 \%$, SP of $97.73 \%$, PPV of $90.00 \%$, and NPV of $93.48 \%$. The AUC estimate for the model was 0.908 (95\% $\mathrm{CI}=0.808-1.008, p<0.001)$. Therefore, the model has an outstanding ability to discriminate. In terms of AUC, the multivariate analysis with three variables (Table 2, Model 2) had the best results with CTP score $>8, \triangle \mathrm{MELD} \geq 1$ and $\triangle \mathrm{CTP} \geq 1$, but only CTP score $>8$ was significantly associated with mortality $(\mathrm{OR}=74.29,95 \% \mathrm{CI}=2.970-1858.49$, $p=0.009)$. The AUC of the prediction rule was 0.941 (95\% CI: $0.862-1.020, p<0.001$ ). In multivariate analysis with all four variables (Table 2, Model 3), only CTP score $>8$ was significantly associated with mortality $(\mathrm{OR}=64.12,95 \% \mathrm{CI}=2.21-1861.08, p=0.015)$. The AUC estimate for the model with all variables was 0.956 (95\% CI $=0.899-1.014, p<0.001)$. The ROC curves for the three considered models (with two, three, and four variables, respectively) indicated that their accuracy was better than that of the models with one variable: $\triangle \mathrm{CTP} \geq 1$ and MELD score $>17$ (Fig. 1).

Models with CTP score $>8$ (as a single factor and combined with $\triangle \mathrm{MELD} \geq 1$ ) had the best SE, SP, PPV, and NPV values. The highest SE and NPV were in the CTP score $>8$ model $(\mathrm{SE}=93.33 \%, \mathrm{NPV}=98.36 \%$; cutoff point $=0.7700)$. The highest SP and PPV were observed in the model with CTP score $>8$ and $\triangle \mathrm{MELD} \geq 1$ ( $\mathrm{SP}=97.73 \%$, PPV $=90.00 \%$, cutoff point $=0.9018)$. Results are shown in Table 3 .

Kaplan-Meier survival curves were estimated for MELD scores $>17(p=0.019)$, CTP scores $>8$ $(p=0.029), \Delta$ MELD $<1(p<0.001)$, and $\Delta$ CTP $<1$ $(p<0.001)$. The higher score values were correlated with a lower survival (Fig. 2).

\section{Discussion}

Both CTP and MELD scales have the ability to discriminate between patients with a higher or lower risk of in-hospital mortality. The MELD score has a predictive value for in-hospital mortality in cirrhotic patients, regardless of liver damage etiology [10, 11]. The CTP score also is known to have predictive significance in short-term and medium-term mortality evaluations in patients with liver cirrhosis [11]. As reported by Samada et al. [12], on comparing CTP to the MELD scale, CTP was found to be more accurate in discriminating patients with liver cirrhosis than the MELD score in

Table 3. Sensitivity, specificity, positive predictive value, and negative predictive value for prediction of mortality determined by logistic regression

\begin{tabular}{lccccc}
\hline \multicolumn{1}{c}{ Variable } & Cutoff point* & SE & SP & PPV & NPV \\
\hline MELD score $>17$ & 0.3056 & 57.89 & 78.99 & 30.56 & 92.16 \\
\hline$\Delta M E L D \geqslant 1$ & 0.5455 & 85.71 & 79.17 & 54.55 & 95.00 \\
\hline CTP score $>8$ & 0.7700 & 93.33 & 60.61 & 26.42 & 98.36 \\
\hline$\Delta C T P \geqslant 1$ & 0.5333 & 80.00 & 82.93 & 53.33 & 94.44 \\
\hline 2 variables (CTP score $>8+\Delta M E L D \geqslant 1$ ) & 0.9018 & 75.00 & 97.73 & 90.00 & 93.48 \\
\hline 3 variables (CTP score $>8+\Delta M E L D \geqslant 1+\Delta C T P \geqslant 1)$ & 0.1550 & 90.00 & 84.62 & 60.00 & 97.06 \\
\hline 4 variables (CTP score $>8+\triangle M E L D \geqslant 1+$ MELD score $>17+\Delta$ CTP $\geqslant 1$ ) & 0.1565 & 90.00 & 84.62 & 60.00 & 97.06 \\
\hline
\end{tabular}

SE - sensitivity; SP - specificity; PPV - positive predictive value; NPV - negative predictive value

${ }^{*}$ Cutoff point means the classification threshold probability. The probability is used to classify patients as surviving (the probability less than this critical value) or non-surviving (the probability equal to or more than the cutoff point) in a logistic regression. 



Fig. 2. Kaplan-Meier survival curves estimated for: A) MELD scores $>17$ and $\leqslant 17$, B) CTP scores $>8$ and $\leqslant 8, C) \Delta M E L D<1$ and $\geqslant 1$, D) $\Delta$ CTP $<1$ and $\geqslant 1$

univariate logistic regression analysis (OR 21.54, AUC 0.770 vs. OR 5.17, AUC 0.684). These two scales are often presented as methods to show the long-term survival rate (where CTP seems to have a more predictive value than MELD); however, there are studies $[12,13]$ suggesting that the MELD score has an advantage over the CTP scale in evaluating short-term survival rate [13]. Nevertheless, it should be noted that the results from this study do not confirm that MELD as a single score has a better discrimination value than CTP. In addition, this study focused on in-hospital (shortterm) observations. Studies that show the insignificance of CTP and MELD model dynamics can also be found. Peeraphatdit et al. [14] found no additional prognostic value of MELD measurements recorded daily for seven days, although it should be noted that their study did not analyze data in the same way as ours. However, it has also been shown that MELD and CTP dynamics (two assessments: first during the admission and the second within a week after admission) were significant. An increase in MELD score by one or more points in univariate logistic regression analysis was the most accurate to determine the in-hospital mortality, and either an increase of MELD score by one point or more or an increase of CTP score by one or more points can have better prognostic value than just the MELD score calculated from admission laboratory tests. 


\section{Conclusions}

This study confirmed that the MELD score could be used to identify patients at a greater risk of death during hospitalization. It also showed that the CTP score could be used to identify patients with greater in-hospital mortality risk. Additionally, the dynamics of these two scores can be used to discriminate patients. An increase in MELD score, even by one point, during hospitalization is significantly associated with a higher mortality rate. The $\triangle \mathrm{CTP}$ was not significant if it was considered as a single indicator. The combined CTP and MELD scores and their kinetics during hospitalization were found to be better predictors of mortality in cirrhotic patients.

\section{Disclosure}

Authors report no conflict of interest.

\section{References}

1. Piotrowski D, Boroń-Kaczmarska A. Bacterial infections and hepatic encephalopathy in liver cirrhosis - prophylaxis and treatment. Adv Med Sci 2017; 62: 345-356.

2. Pugh RN, Murray-Lyon IM, Dawson JL, et al. Transection of the oesophagus for bleeding oesophageal varices. Br J Surg 1973; 60: 646-649.

3. Bambha K, Kim WR, Kremers K, et al. Predicting survival among patients listed for liver transplantation: an assessment of serial MELD measurements. Am J Transplant 2004; 4: 17981804.

4. StatSoft, Inc. (2014). STATISTICA (data analysis software system), version 12. www.statsoft.com

5. R Development Core Team. R: a language and environment for statistical computing, R Foundation for Statistical Computing, Vienna, Austria, 2015. Available from: http://www.r-project.org

6. Warnes GR, Bolker B, Lumley T, et al. Gmodels: various R programming tools for model fitting. 2015. Available from: https:// cran.r-project.org/web/packages/gmodels/index.html

7. Lopez-Raton M, Rodriguez-Alvarez MX. OptimalCutpoints: computing optimal cutpoints in diagnostic tests. 2015. Available from: https://cran.rproject.org/web/packages/OptimalCutpoints/index.html

8. Therneau TM, Lumley T. Survival: A package for survival analysis in S, R Package, version 2.37-7. Available from: http:// cran.r-project.org/package $=$ survival

9. NCAR - Research Applications Laboratory. Verification: Weather forecast verification utilities. 2015. Available from: https:// cran.r-project.org/web/packages/verification/index.html

10. Kraja B, Sina M, Mone I, et al. Predictive value of the Model of End-Stage Liver Disease in cirrhotic patients with and without spontaneous bacterial peritonitis. Gastroenterology Research and Practice 2012, Article ID 539059, doi: 10.1155/2012/539059

11. Cheng XP, Zhao J, Chen Y, et al. Comparison of the ability of the PDD-ICG clearance test, CTP, MELD, and MELD-Na to predict short-term and medium-term mortality in patients with decompensated hepatitis B cirrhosis. Eur J Gastroenterol Hepatol 2016; 28: 444-448.
12. Samada M, Hernández JC. Prognostic Factors for survival in patients with liver cirrhosis. In: Prof. Hesham Abdeldayem (ed.). Liver Transplantation - Basic Issues, ISBN: 978-953-51-0016-4, InTech; 2012; 125-144. Available from: http://www.intechopen. com/books/liver-transplantation-basic-issues/prognostic-factors-for-survival-in-patients-with-liver-cirrhosis

13. Šumskienė J, Kupčinskas L, Pundzius J, et al. Prognostic factors for short and long-term survival in patients selected for liver transplantation. Medicina (Kaunas) 2005; 41: 39-46.

14. Peeraphatdit T, Naksuk N, Thongprayoon C, et al. Model for End-Stage Liver Disease (MELD) score measurements on the daily basis in critically ill cirrhotic patients do not provide additional prognostic value. Mayo Clin Proc 2015; 90: 1196-1206. 